\title{
Anforderungen an die Qualifikation und die Überwachung von nichtärztlichem Personal im Strahlenschutz
}

\section{- Anmerkung zum Urteil des VGH Baden-Württemberg vom 17.12.2012}

Der technische Leistungsanteil in der bildgebenden Diagnostik, wie etwa beim Röntgen und der Computertomografie, werden als delegierbare ärztliche Leistungen angesehen, deren Durchführung der Radiologe nach der Anlage 24 zum Bundesmantelvertrag-Ärzte - BMV-Ä (vgl. DÄBl. 2013, A 1757) auf entsprechend qualifizierte nichtärztliche Mitarbeiter übertragen kann (vgl. hierzu Fortschr Röntgenstr 2013; 185, 1213). Über welche Qualifikation das technisch mitwirkende Personal verfügen muss und in welchem Umfang dieses durch den Arzt überwacht bzw. beaufsichtigt werden muss, ergibt sich aus den Bestimmungen in der Strahlenschutzverordnung (StrlSchV) und der Röntgenverordnung (RöV) sowie aufgrund der Richtlinie Strahlenschutz in der Medizin.

Der Verwaltungsgerichtshof (VGH) Baden-Württemberg hat sich mit Urteil vom 17.12.2012 (Az.: 10 S 1340/12) mit den
Anforderungen der Aufgabenübertragung in der Strahlentherapie auseinandergesetzt und der Möglichkeit einer Delegation Grenzen gesetzt. Das Urteil ist jedoch nicht nur für das Fachgebiet der Strahlentherapie, sondern auch für die Radiologie von Bedeutung, weil der VGH die Auffassung vertreten hat, dass an die ständige Aufsicht und Verantwortung nach der RöV keine geringeren Anforderungen als im Rahmen der StrlSchV gestellt werden.

Soweit man einer Übertragbarkeit der Grundsätze des Urteils auf die Radiologie folgt, sind die Ausführungen insbesondere für folgende Fragen von Bedeutung:

1. Anforderungen an die Qualifikation des technisch mitwirkenden Personals nach § 24 Abs. 2 RöV,

2. Räumliche Anforderungen an die „ständige Aufsicht und Verantwortung" des Radiologen nach $\S 24$ Abs. 1 Satz 1 Nr. 3 und Abs. 2 RöV.

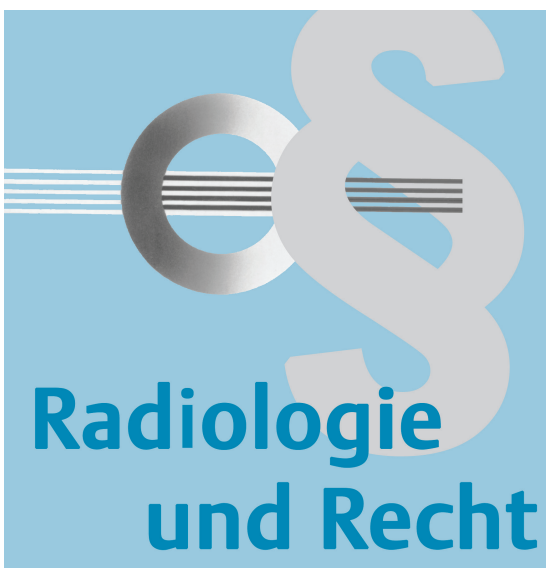

\section{Sachverhalt}

In dem von dem VGH zu beurteilenden Sachverhalt ging es um die Änderung einer Genehmigung zum Betrieb des Linearbeschleunigers Tomotherapie HiArt in einer aus 2 Fachärzten für Strahlentherapie bestehenden Gemeinschaftspraxis. Die Genehmigungsbehörde für den Linearbeschleuniger erteilte die entsprechende Betriebsgenehmigung unter der Auflage, dass der Beschleuniger in der Regel von 2 fachkundigen medizinischtechnischen Radiologieassistenten (MTRA) zu bedienen sei, die die Lagerung des Patienten, die Eingabe der Bestrahlungsdaten, das Einschalten der Strahlung 
und die Überwachung der Behandlung am Schaltpult durchführen. Im Falle der Abwesenheit eines MTRA solle, so das Regierungspräsidium, der für die Therapie zuständige Arzt bei der Patentenlagerung bzw. der Patienteneinstellung helfen. Zudem seien die in der „Richtlinie Strahlenschutz in der Medizin“ (Fassung vom 24.06.2002) aufgestellten Forderungen zu erfüllen.

Keine Berücksichtigung der Richtlinie Strahlenschutz in der Medizin $\nabla$

Bemerkenswert ist zunächst, dass das Gericht zur Beurteilung der Rechtslage nicht auf die Richtlinie zur Verordnung über den Schutz vor Schäden durch ionisierende Strahlen - Richtlinie Strahlenschutz in der Medizin - vom 17.10.2011 (GMBl. 2011, S. 867) des Bundesministeriums für Umwelt, Naturschutz und Reaktorsicherheit, die der Länderausschusses für Atomkernenergie erarbeitet hat, abstellt. Die Richtlinie sei weder eine Rechtsnorm noch eine - normkonkretisierende - Verwaltungsvorschrift. Es fehle schon die notwendige gesetzliche Ermächtigungsgrundlage. Zudem sei zu berücksichtigen, dass ihre Vorgaben nicht der Strahlenschutzverordnung entsprächen und daher hinter diese Rechtsnorm zurücktreten müsse. Bedeutsam sei allein, dass $\S 14$ Abs. 1 Satz 1 Nr. 6 der Strahlenschutzverordnung (StrlSchV) verlangt, dass keine Tatsachen vorliegen, aus denen sich Bedenken ergeben, dass das für eine sichere Ausführung des Betriebes notwendige Personal nicht vorhanden ist. Für das Gericht ist somit allein die Strahlenschutzverordnung für die Beurteilung des Sachverhalts entscheidend. Sollte sich diese Rechtsauffassung durchsetzen bzw. vom Bundesverwaltungsgericht bestätigt werden, dürfte es erforderlich sein, für die Richtlinie Strahlenschutz in der Medizin eine ausreichende Ermächtigungsgrundlage in der StrlSchV und der RöV zu schaffen, da auf deren normkonkretisierende Regelungen nicht ohne weiteres verzichtet werden kann.

\section{Vier-Augen-Prinzip \\ $\nabla$}

Der VGH führt aus, dass in der Strahlentherapie die in $\S 82$ Abs. 2 StrlSchV genannten Personen technisch mitwirken dürfen, also Handlungen ausführen dürfen, die der praktischen Durchführung der vom Arzt verantworteten Therapie die- nen, worunter auch die in diesem Rechtsstreit zu beurteilenden Maßnahmen fallen.

Dabei reiche es im Bereich der Strahlentherapie für einen sicheren Betrieb nicht aus, wenn nur eine Person die Handlungen vornehme. Das geforderte notwendige Personal sei so zu bemessen, dass der sichere Betrieb der Anlage gewährleistet sei, insbesondere, dass jede unnötige Strahlenexposition vermieden und jede Strahlenexposition gemäß $§ 6$ i.V.m. § 2 Abs. 1 Nr. $1 \mathrm{~d}$ StrlSchV so gering wie möglich gehalten wird. Zwar sei ein Betrieb des Linearbeschleunigers grundsätzlich auch mit einer Person technisch möglich, allerdings richte sich der Personaleinsatz nach einer individuellen Betrachtung auf der Grundlage des Sicherheitskonzeptes des Betreibers, wobei sich die Personenzahl nach dem Risikopotential richtet. In Anbetracht der erheblichen Risiken für Leben und Gesundheit, die mit einer fehlerhaften Bestrahlung verbunden sind, sind nach Ansicht des Gerichts für den sicheren Betrieb im Normalfall mindestens 2 Personen erforderlich, die bei der Durchführung der Bestrahlung technisch mitwirken müssen, um sich gegenseitig zu unterstützen und zu ergänzen.

Die Vermeidung unnötiger Strahlenexposition findet sich auch in $\S 2 c$ RöV. Dennoch ist die Forderung nach einer technischen Mitwirkung von mindestens 2 Personen auf die Durchführung von Maßnahmen nach der RöV nicht übertragbar, da ein vergleichbares Risikopotential für den Patienten aufgrund der Strahlendosis im Bereich der Röntgenverordnung nicht gegeben ist. Im Übrigen spielen im Bereich der Radiologie Probleme wie die genaue Positionierung des Patienten und entsprechende Folgen bei Konzentrationsfehlern des Personals keine vergleichbare Rolle. Die Forderung nach einem grundsätzlichen „Vier-Augen-Prinzip“ ist daher auf radiologische Leistungen, die der RöV unterfallen, nicht übertragbar.

\section{Qualifiziertes medizinisch-techni- sches Fachpersonal \\ $\nabla$}

Übertragbar auf die Radiologie sind, jedenfalls teilweise, die Ausführungen des Gerichts zu den Anforderungen an das qualifizierte medizinisch-technische Fachpersonal nach $\S 82$ Abs. 2 StrlSchV, die bei der Anwendung technisch mitwirken dürfen.
Zur „technischen Mitwirkung“ ist nach § 82 Abs. 2 Nr. 1 und 2 StrlSchV nur qualifiziertes medizinisch-technisches Fachpersonal befugt, welches über die Fachkunde im Strahlenschutz verfügt. Die Fachkunde wird erworben durch eine für den jeweiligen Anwendungsbereich geeignete Ausbildung, praktische Erfahrung und die erfolgreiche Teilnahme an anerkannten Kursen (§ 30 Abs. 1 Satz 1 StrlSchV). Nach dem Konzept der Strahlenschutzverordnung setzt der Erwerb der Fachkunde durch nichtärztliches Personal eine abgeschlossene Berufsausbildung der in $\S 82$ Abs. 2 Nr. 1 und Nr. 2 StrlSchV genannten Art voraus.

Diese Anforderungen sind in der RöV in identischer Form geregelt, wobei § 24 Abs. 2 Satz 1 RöV den Begriff der „technischen Durchführung“ verwendet, der die Eigenständigkeit der Leistungserbringung durch die genannten Personen daher bereits stärker betont. Auch nach $\S 24$ Abs. 2 Nr. 1 oder 2 RöV ist zur technischen Durchführung radiologischer Untersuchungen nur qualifiziertes medizinisch-technisches Fachpersonal berechtigt, namentlich entweder gemäß § 24 Abs. 2 Nr. 1 RöV eine Person „mit einer Erlaubnis nach $\S 1$ Nr. 2 des MTA-Gesetzes“ (Medizinischtechnische Radiologieassistentin oder Medizinisch-technischer Radiologieassistent; im Folgenden: MTRA) oder gemäß § 24 Abs. 2 Nr. 2 RöV eine Person „mit einer staatlich geregelten, staatlich anerkannten oder staatlich überwachten abgeschlossenen Ausbildung, wenn die technische Durchführung Gegenstand ihrer Ausbildung und Prüfung war und sie die erforderliche Fachkunde im Strahlenschutz" besitzt (i.d.R. Medizinisch-technische Assistentin oder Medizinischtechnischer Assistent mit entsprechender Zusatzqualifikation und Fachkunde im Strahlenschutz gemäß $§ 18$ a Abs. 1 und 2 RöV; im Folgenden: MTA mit Zusatzqualifikation).

Personen, die, wie medizinische Fachangestellten (MFA), die lediglich über eine sonstige medizinische Ausbildung im Sinne des § 82 Abs. 2 Nr. 4 StrlSchV bzw. § 24 Abs. 2 Nr. 4 RöV verfügen, können die Fachkunde dagegen nicht erwerben. Die unter Nr. 4 genannten Personen verfügen lediglich über eine sonstige medizinische Ausbildung und die erforderlichen Kenntnisse im Strahlenschutz. Die erforderlichen Kenntnisse werden in der Regel durch eine für das jeweilige Anwendungsgebiet geeignete Einweisung und prakti- 
sche Erfahrung erworben ( $\$ 30$ Abs. 4 Satz 1 StrlSchV, § 18a Abs. 3 Satz 1 RöV) und bleiben daher hinter der Fachkunde zurück. Die fehlende Fachkunde bei Personen nach $\S 82$ Abs. 2 Nr. 4 StrlSchV bzw. $\S$ 24 Abs. 2 Nr. 4 RöV wird dadurch kompensiert, dass diese Personen nur „unter ständiger Aufsicht und Verantwortung" eines fachkundigen Arztes tätig werden dürfen. Den mit bestimmten Tätigkeiten verbundenen Risiken ist durch eine entsprechend intensive Aufsicht des fachkundigen Arztes zu begegnen.

Während einer durchgeführten Röntgenuntersuchung hat der anwesende, verantwortliche Radiologe daher die Wahl, die Untersuchung technisch entweder selbst durchzuführen ( $\S 24$ Abs. 2 i.V.m. Abs. 1 RöV) oder einem qualifizierten medizinisch-technischen Fachpersonal (MTRA oder MTA mit Zusatzqualifikation) zur selbständigen technischen Durchführung zu übertragen ( $\S 24$ Abs. 2 Nr. 1 oder 2 RöV) oder aber unter seiner ständigen Aufsicht und Verantwortung entweder Ärzte ohne entsprechende Fachkunde, aber mit entsprechenden Kenntnissen im Strahlenschutz ( $\S 24$ Abs. 2 i.V.m. Abs. 1 Nr. 3 RöV) oder Personen mit einer abgeschlossenen sonstigen medizinischen Ausbildung mit den erforderlichen Kenntnissen im Strahlenschutz ( $§ 24$ Abs. $2 \mathrm{Nr}$. 4 RöV) oder Auszubildende im fachlich einschlägigen Bereich (§ 24 Abs. 2 Nr. 3 RöV) unselbstständig einzusetzen.

Zwar dürfen Medizinische Fachangestellte daher ohne Einschränkungen hinsichtlich der technischen Durchführung wie Ärzte oder MTRA tätig werden. Allerdings dürfen sie nur unter ständiger Aufsicht und Verantwortung eines fachkundigen Arztes tätig werden.

\section{Begriff der „ständigen Aufsicht ${ }^{\text {“ }}$}

Diese Kompensationsfunktion ist dann auch der Maßstab dafür, wie weit die ständige Aufsicht und Verantwortung des fachkundigen Arztes reichen muss. Die vom VGH aufgestellten Anforderungen an die Aufsichts- und Überwachungspflichten sind sehr intensiv und gehen über die Anforderungen nach der Richtlinie Strahlenschutz in der Medizin deutlich hinaus bzw. widersprechen der Richtlinie sogar. Zu prüfen ist allerdings, ob, wie der VGH meint, die Anforderungen nach der StrlSchV auf die RöV übertragbar sind, da die gesetzlichen Voraussetzungen in $§ 82$
Abs. 2 Nr. 4 StrlSchV und § 24 Abs. 2 Nr. 4 RöV vom Wortlaut in gleicher Art und Weise geregelt sind.

Das Gericht führt zunächst aus, dass an die ständige Aufsicht und Verantwortung im Sinne der Nrn. 3, 4 und 5 des $\S 82$ Abs. 2 StrlSchV jeweils unterschiedliche Anforderungen zu stellen seien, da die zu beaufsichtigenden Personen unterschiedliche Qualifikationen aufweisen. Im Bereich der hier maßgeblichen Nr.4 StrlSchV verfügen diese Personen über eine sonstige medizinische Ausbildung. Die ständige Aufsicht und Verantwortung muss die fehlende Fachkunde der Personen nach $\S 82$ Abs. 2 Nr. 4 StrlSchV daher kompensieren. Diese unterschiedlichen Aufsichtsanforderungen gelten in der Radiologie entsprechend, da § 24 Abs. 2 Nr. 3, 4 und 5 RöV auf die gleichen Personengruppen mit unterschiedlichen Qualifikationen verweist. Die Intensität der Aufsicht könne zudem je nach Art der technischen Mitwirkung und den damit verbundenen Risiken variieren.

Der Wortlaut „ständig“ bringe zum Ausdruck, dass die Aufsicht fortlaufend erfolgen müsse. Eine auf Stichproben beschränkte Kontrolle sei damit jedenfalls dann nicht zu vereinbaren, wenn es sich wie im Bereich der Strahlentherapie um Tätigkeiten handele, die mit erheblichen Risiken für die Gesundheit des Patienten verbunden sind. Ob diese Anforderungen einer fortlaufenden Überwachung von Personen ohne Fachkunde im Strahlenschutz nach § 24 Abs. 2 Nr. 4 RöV auch bei radiologischen Untersuchungsleistungen in dem gleichen Umfang gelten, ist angesichts des mit einer Röntgenuntersuchung verbundenen geringeren Risikopotentials zu hinterfragen.

Der VGH ist der Auffassung, dass an die ständige Aufsicht und Verantwortung nach § 24 Abs. 2 Nr. 4 RöV keine geringere Anforderungen als vorliegend im Rahmen des $\S 82$ Abs. 2 Nr. 4 StrlSchV gestellt werden könnten. Der VGH verweist auf die Rechtsprechung des Bayerischen Verwaltungsgerichtshofs (Urteile vom 14.04.2008, Az.: 9 B 08.80; 9 B 08.81; 9 B 08.94), nach der der Begriff der ständigen Aufsicht und Verantwortung im Sinne der Röntgenverordnung verlange, dass sich der verantwortliche Radiologe in unmittelbarer Nähe aufhalten und die Tätigkeit laufend überwachen müsse, um erforderlichenfalls jederzeit korrigierend eingreifen zu können. Bei einer Röntgenbehand- lung gebiete es darüber hinaus $\S 27$ Abs. 2 Nr. 2 RöV, dass - unabhängig von der Qualifikation der mit der technischen Durchführung befassten Personen - die Einhaltung aller im Bestrahlungsplan festgelegten Bedingungen vor Beginn jeder Bestrahlung von einem Arzt mit der erforderlichen Fachkunde überprüft werde.

Ausdrücklich reicht es nach Ansicht des VGH nicht aus, dass der Arzt nur jederzeit verfügbar sei, um bei während der Behandlung auftretenden Problemen helfen bzw. auf Fragen des Patienten eingehen zu können. Erst recht reiche es nicht aus, dass der fachkundige Arzt, wie es die Richtlinie Strahlenschutz in der Medizin 2011 in den Begriffsbestimmungen in Anlage B 10 vorsehe, innerhalb von 15 Minuten herbeigerufen werden könne. Mit dem Zweck der ständigen Aufsicht, die fehlende Fachkunde der zu beaufsichtigenden Person zu kompensieren, sei es nicht zu vereinbaren, dass die zu beaufsichtigende Person die Aufsicht durch Herbeirufen des Arztes einfordern müsse, auch wenn dies angesichts der Gegebenheiten in der Praxis binnen Sekunden möglich wäre. Es dürfe nicht von der Entscheidung der zu beaufsichtigenden Person abhängen, wann eine Aufsicht stattfinde.

Jedenfalls bei den risikoreichen Tätigkeiten der technischen Mitwirkung bei einem Bestrahlungsvorgang nach der StrlSchV sei es erforderlich, dass der fachkundige Arzt die Person nach $\S 82$ Abs. 2 Nr. 4 StrlSchV laufend überwache und jederzeit korrigierend eingreifen könne. Damit geht der VGH jedoch selbst davon aus, dass es unterschiedliche Überwachungsqualitäten aufgrund der unterschiedlichen Risiken bei Strahlenbehandlungen und der Diagnostik geben kann. Der Umstand, dass es sich bei der Durchführung von Untersuchungen nach der RöV um Eingriffe mit einer geringeren Eingriffsintensität mit einem deutlich geringeren Risikopotential für die Patienten handelt, ist angesichts der Tatsache, dass in der Strahlentherapie bewusst eine hohe Strahlendosis eingesetzt wird, um Tumorzellen zu schädigen, einleuchtend. Aufgrund dieser Tatsache führen Fehlbestrahlungen auch zu einer unmittelbaren Schädigung des Patienten, während in der radiologischen Diagnostik das Gefährdungspotential des Patienten durch eine fehlerhafte Untersuchung deutlich geringer anzusetzen ist. 
Zwar ist dem VGH zuzugestehen, dass auch bei Röntgenuntersuchungen vermeidbare Strahlenbelastungen für Patienten, Personal und Dritte dadurch auftreten können, dass das Röntgengerät unsachgemäß bedient wird oder Fehlfunktionen des Geräts nicht rechtzeitig erkannt oder nicht fachlich kompetent abgestellt werden. Auch sind unbrauchbare Untersuchungen mit der Folge von Doppeluntersuchungen denkbar. Allerdings ist davon auszugehen, dass diese Risiken für das Fachgebiet der Radiologie regelmäßig durch den Erwerb der erforderlichen Kenntnisse im Strahlenschutz nach § 18a Abs. 3 RöV durch eine für das jeweilige Anwendungsgebiet geeignete Einweisung und praktische Erfahrung erworben werden, sodass bei einer eingewiesenen und erfahrenen MFA kein entsprechendes Risiko besteht, sodass es ausreichend ist, wenn sich der Radiologe in erreichbarer Nähe zur Untersuchung aufhält.

Für die sog. Röntgenbehandlung nach § 27 RöV, die wie die Strahlentherapie eine therapeutische Bestrahlung zum Gegenstand hat, erscheint es demgegenüber angemessen, die gleichen Anforderungen an die ständige Aufsicht anzulegen, wie in der Strahlentherapie selbst.

Die Richtlinie Strahlenschutz in der Medizin, die für die Strahlentherapie Anwendung findet, versucht die Überwachungspflichten, entsprechend dem Verhältnismäßigkeitsgrundsatz, dem Risiko der Behandlung anzupassen. In den Begriffsbestimmungen in der Anlage B 10 der Richtlinie Strahlenschutz in der Medizin 2011 wird unterschieden zwischen „unmittelbarer Aufsicht“, die in direkter räumlicher Nähe des zu Beaufsichtigenden stattfindet, womit ein sofortiges Eingreifen bei einer eventuellen Fehlhandlung möglich ist und einer „ständigen Aufsicht“, unter der die Erreichbarkeit in einem Zeitraum von nicht mehr als 15 Minuten zu verstehen sei. Der VGH erkennt diese unterschiedlichen Anforderungen an die Beaufsichtigung in der Richtlinie Strahlenschutz in der Medizin jedoch nicht an, da der Begriff der „unmittelbaren Aufsicht“ in der Strahlenschutzverordnung keine Stütze finde. Die Strahlenschutzverordnung verwende den Begriff der unmittelbaren Aufsicht nicht und nehme daher auch keine Differenzierung zwischen unmittelbarer und ständiger Aufsicht vor.

\section{Fazit und Ausblick für die Praxis $\nabla$}

Die Argumentation des Verwaltungsgerichtshofs Baden-Württemberg ist für die Strahlentherapie größtenteils nachvollziehbar und angemessen. Hinsichtlich des Einsatzes von Personen ohne die erforderliche Fachkunde im Strahlenschutz nach $\S$ 82 Abs. 2 Nr. 4 StrlSchV verlangt das Gericht zutreffend eine Beaufsichtigung in direkter räumlicher Nähe durch den fachkundigen Arzt. Hinsichtlich der Auslegung des Erfordernisses der ständigen ärztlichen Aufsicht von Personen ohne Fachkunde im Strahlenschutz bei der technischen Durchführung dieses Personenkreises bleibt festzuhalten, dass eine lückenlose Überwachung, die in die Nähe einer permanenten Anwesenheit des Strahlentherapeuten während der Behandlung rückt, angesichts des Risikopotenzials dieser Behandlungsmethode nachvollziehbar erscheint.

Dagegen ist die vom Gericht „en passant“ vorgenommene Übertragung dieser Grundsätze auf den Regelungsbereich der RöV, trotz des vergleichbaren Wortlauts, nicht zwingend und konsequent, vor allem wenn man das Risikopotenzial in der diagnostischen Radiologie berücksichtigt. Der VGH räumt einerseits selbst ein, dass die Intensität der Aufsicht je nach Art der technischen Mitwirkung und den damit verbundenen Risiken variieren könne, stellt jedoch an anderer Stelle relativ undifferenziert fest, dass nicht ersichtlich sei, ,dass an die ständige Aufsicht und Verantwortung im Sinne der Röntgenverordnung geringere Anforderungen“" als nach $\S 82$ Abs. 2 Nr. 4 StrlSchV bei dem Einsatz von Personal ohne Fachkunde im Strahlenschutz zu stellen sei. Dabei ist die Notwendigkeit einer unterschiedlichen Auslegung des Begriffs der „ständigen Aufsicht" in $\S 82$ Abs. 2 Nr. 4 StrlSchV einerseits und $\S 24$ Abs. 2 Nr. 4 RöV andererseits, angesichts des unterschiedlichen Risikopotenzials beider Verfahrensarten ohne Weiteres einleuchtend. Dies gilt jedenfalls für die technischen Verfahren der diagnostischen Radiologie, während für die Röntgentherapie nach $\S 27$ RöV die gleichen Anforderungen wie in der Strahlentherapie zu stellen sind.

Diesem Anliegen trägt der Begriff in Anlage B 10 der Richtlinie Strahlenschutz in der Medizin 2011 Rechnung, in dem dort der Begriff der Aufsicht in unmittelbare und ständige unterteilt wird. Für die diagnostische Radiologie ist es demnach ausreichend, dass eine ständige Aufsicht im Sinne einer Erreichbarkeit in einem kurzen Zeitraum durch den Arzt gegeben ist.

Angesichts der durch die Ausführungen des VGH entstandenen unklaren Rechtslage bzgl. der Intensität der Beaufsichtigung des Personenkreises nach § 24 Abs. 2 Nr. 4 RöV und der Tatsache, dass das Gericht die Richtlinie Strahlenschutz in der Medizin 2011 für nicht im Einklang mit den Vorgaben der StrlSchV hält, ist der Gesetz- und Verordnungsgeber aufgerufen, sowohl in der StrlSchV, als auch in der RöV, eine Konkretisierung des unbestimmten Rechtsbegriffs der „ständigen Aufsicht“ aus Gründen der Sicherheit für die betroffenen Patienten und Ärzte vorzunehmen.

\section{RA Dr. Peter Wigge \\ Fachanwalt für Medizinrecht}

Lic. iur. can. Urs Fabian Frigger

Rechtsanwalt

Rechtsanwälte Wigge

Scharnhorststr. 40

48151 Münster

Telefon: (0251) 53595-0

Telefax: (0251) 53595-99

E-Mail: kanzlei@ra-wigge.de www.ra-wigge.de 